\title{
The Effect of a Self-Management Program Combined with Social Support on the Self-Management and Healthy Eating Behaviors among Patients with Cardiovascular Disease
}

\section{Pachanut NUNTHAITAWEEKUL* and Junpen PANSUP}

\author{
Faculty of Nursing, Chulalongkorn University, Bangkok 10330, Thailand
}

("Corresponding author's e-mail: pachanut.t@chula.ac.th)

Received: 5 March 2020, Revised: 13 September 2020, Accepted: 30 October 2020

\begin{abstract}
Unhealthy eating behaviors are one of the major causes of Coronary vascular disease (CVD). The self-management program combined with social support (CIP) was implemented to improve the patients' self-management ability and maintain their eating behaviors to a healthier standard. The purpose of this study was to compare the scores of self-management and healthy eating behaviors of patients with CVD in the experimental CIP group before and after participation in the CIP. It also compared selfmanagement and healthy eating behavior scores between patients with CVD who entered the CIP with the usual nursing care group. This study was a quasi-experimental approach with 2 groups of pre-and posttests. The sample size of 50 cases, 25 in each group, was considered for the study. Questionnaires were used to assess the patient's self-management and healthy eating behavior scores. Descriptive statistics, dependent t-test, and independent t-test were used for the data analysis. The findings revealed that the experimental group showed a significantly $(\mathrm{p}<0.05)$ increased average self-management $(46.2 \pm 5.6$ to $51.1 \pm 5.9)$ and healthy eating behaviors $(39.3 \pm 5.5$ to $48.4 \pm 4.0)$ scores after the program than before. They also had a significantly $(\mathrm{p}<0.05)$ higher self-management and healthy eating behavior scores than the control group $(51.1 \pm 5.9$ vs. $48.9 \pm 6.0$ and $48.4 \pm 4.0$ vs. $39.8 \pm 7.5$, respectively). This program increased the patients' self-management scores, and consequentially their healthy eating behaviors score also improved.
\end{abstract}

Keywords: The self-management program, Social support, Cardiovascular disease, Self-management, Healthy eating behaviors

\section{Introduction}

Coronary heart disease (CHD), which is the leading cause of death among people worldwide, is a major problem. The global mortality rate is seen to increase to 23 million people in 2030 [1]. There were more than 785,000 new myocardial infarctions (MI) each year in the USA, of which more than 232,000 were treated by a coronary artery bypass graft [2]. Coronary vascular disease (CVD) refers to heart disease caused by abnormal coronary arteries, which causes the insufficient blood supply to the heart muscle, leading to myocardial ischemia, MI, and heart failure [3-6].

One of the major causes that increase complications in cardiovascular disease (CVD) is inappropriate (unhealthy) eating behaviors, or eating habits, a lack of a balanced diet, lifestyle changes, such as eating food with high cholesterol, triglycerides, salt and sugar contents, a low fiber content, and drinking excessive alcohol or caffeine [6-11]. These types of food will tend to cause fat accumulation and a high serum level of both cholesterol and low-density lipoproteins that can build up around the blood vessels' walls (plaque). When there is long term accumulation, the blood vessels become narrower. If the plaque ruptures, it can cause a partial or total blockage of the blood vessel, which prevents the blood from 
flowing through the tissues. In the case of the heart, this can lead to sudden ischemia, infarction, and heart failure $[10,12,13]$. The important factors that make patients commit to inappropriate (unhealthy) eating behaviors or eating habits is the lack of knowledge, misunderstanding about CVD, and dietary behavior changes. People thought that taking medication was the only way to get rid of this disease. They also do not have the confidence to be conscious of their diet behaviors. Therefore, encouraging patients to selfmanagement is a very good form of self-care. $[4,8,9,12]$.

Self-management helps patients with CVD adjust their eating behaviors to be better balanced (healthier) and more suited to their conditions to prevent a recurrence and control the severity of the CVD symptoms [9-13]. Self-management is self-acting control of the illness and a behavior modification process [14]. As such, self-management consists of (i) goal setting, (ii) data collection, (iii) data analysis and evaluation, (iv) decision making, (v) action, and (vi) post-performance evaluation. Although selfmanagement may help people's eating behaviors, it cannot maintain for a long time because patients with CVD must control risk behaviors for the long life, social support was necessary. Social support consists of passion, care, trust, financial help, things, labor, and informational provision, including providing feedback for self-assessment and information for learning [15]. A combined intervention program (CIP) was adopted in this study to develop and maintain a healthy eating behavior. The CIP combined a selfmanagement program with social support at the 3 support dimensions of emotional, tangible, and information support. The emotional dimension includes expressions of caring, empathy, and acceptance. The tangible aid is related to daily life activities, such as transport, cooking, and relaxation. The informational support includes advice and suggestions on what to do, managing problems, and responding to personal needs [14]. The social support comes from the family, spouse, and personnel in the health system. An effective social support group will motivate one another to engage in healthy eating behaviors and continue for long-term care [16-18].

Previous studies found that self-management of social support has a positive impact on improving patient behavior. However, people cannot maintain for the long term; thus, most patients still have the same eating behavioral problem. Therefore, the researcher applies two concepts, self-management and social support, to improve the program. Using the two concepts makes this study different from previous studies.

Social support refers to receiving various support from close friends and loved ones, medical staff personnel, and service sources in the community [19-21]. Therefore, social support should be involved in the process of self-management that is essential for all patients. Furthermore, the health provider constantly needs to follow-up the patience from the hospital to the community. This practice could encourage and maintain the behavior that will continue to impact their daily routine positively. Finally, the reoccurrence and hospital readmission rates will also be reduced accordingly.

This study aimed to (i) to compare the scores of self-management and healthy eating behaviors of patients with CVD in the experimental CIP group (self-management program combined with social support) before and after participation in the CIP. It also compared the self-management and healthy eating behavior scores between patients with CVD who entered the CIP with the group receiving and the usual nursing care.

\section{Materials and methods}

This study was a quasi-experimental research with 2 groups of a pre- and a post-test non-equivalent control group design.

\section{Study procedure}

The sample size was calculated by controlling the occurrence of type 2 errors and determining the test power of $80 \%$, the significance level of 0.05 , and the influence size of 0.05 [22]. This yielded 44 cases by random sampling. However, the sample size was increased by $10 \%$ to allow for potential dropouts from the study [23]. Therefore, the sample group used in this study was 50 people, 25 per group.

Inclusion criteria 1) the participants (study subjects) were male and female patients with CVD, 2) having treated for medication or PCI or CABG, 3) having a stable heart disease (clinical sign and 
symptom), 4) aged 40 - 65 years old, 5) can communicate by speaking, reading, and writing in Thai, 6) have family to join the program at any time. Exclusion criteria 1) experiencing side effects and must be admitted in the hospital, 2) participant and family could not join the program with 8 weeks.

\section{Data collection}

1. Researcher and research assistant study the patients' profile according to the specified qualifications and met participants who came to receive services at the outpatient department, heart disease clinic, Bang Nam Priao hospital, Chachoengsao province, Thailand. Match paired with gender and age (each couple is not more than 5 years) was adopted in this study for control of other variables that may influence the combined dependent variable.

2. The data collection in the control group was finished first before proceeding with the experimental group to prevent contamination of data. The outline below shows the process.

Control group:

At the start (week 0), the patients were met at the cardiology clinic (30 min) to clarify the project objectives. Following explanation of the ethical protocol, the patients were asked to complete the demographic and illness questionnaire, and then the self-management assessment and healthy eating behavior questionnaires (pre-test). Patients could ask questions and receive self-care as usual. Appointments were made for 6 weeks later.

After 6 weeks, the patients were asked to complete the self-management assessment and healthy eating behavior questionnaires (post-test). After that, the patients were admitted into the CIP, the same as the experimental group (see below), and recommended for examination by appointment at the hospital.

Experimental (CIP) group:

At the start (week 0) the patients and family were met at the cardiology clinic (30 min) to clarify the project objectives and were then asked to answer both questionnaires (pre-test), the same as the control group (above). In addition, the patients and family were provided from CIP that includes: Part I) basic knowledge on CVD (pathos-physio of CVD, cause of CVD, treatment, caring), heart-healthy diets, nutrition therapy, and food selection science, while social support from relatives, caregivers, medical personnel, sources or health service systems, were also provided. The patients were made aware of the right to treatment and continuous care policy system $(30 \mathrm{~min})$. Part II) the patients received knowledge and skills in organizing specific menu items for CVD (20 min) and break time (15 min). Part III) these self-management skills consisted of steps as detailed in the program (20 min). The patients were supplied a diet guide on CVD to read and review as well as self-management records for patients with CVD.

Then, a $2^{\text {nd }}$ (follow-up) contact in the $2^{\text {nd }}$ and $4^{\text {th }}$ week provided the patients in the CIP group further stimuli for self-management. Questions about various problems and records of self-management practice were administered using a follow-up phone call, which took 15 - $20 \mathrm{~min}$ by a primary care nurse and the research team. If the participants had problem, they would be asked how they solve the problem. This information was also added to help manage their self-management plan.

In week 5, a follow-up meeting with each patient as a home visit was performed to evaluate the patient's self-management records and assess their self-management ability. An appointment for the evaluation of the patient's diet behavior by the research team and primary care nurse was also made, which took about 30 - 45 min, depending on the patient's problem.

Finally, in week 6, the patients were asked to complete the evaluation of self-management assessment and healthy eating behavior questionnaires (post-test) by the research team and primary care nurse, which took $30 \mathrm{~min}$. This then, concluded the data collection. The patients were recommended for examination by making an appointment at the hospital.

\section{Materials}

(i) Demographic and illness questionnaire. The questionnaire for patients with CAD was created by the authors and consisted of items about gender, age, marital status, occupation, comorbidity, heightweight and body mass index (BMI), and history of treatment.

(ii) Self-management assessment form. This was formed by translating the existing questionnaire into Thai language after adjusting the questions to be suitable for the sample group from [24]. The patient 
activation measure (PAM) questionnaire and faculty questions were translated into Thai [25] and consisted of 13 questions (duration of score 13 - 52), all of which had a positive meaning. The questionnaire was found to have a Cronbach's alpha coefficient equal to 0.80 using a 4 level rating scale.

(iii) Questionnaire for healthy eating behavior. This was adapted in-house from the heart healthy eating questionnaire [26] using the existing Thai version [27], and consisted of 16 questions (duration of score 16 - 64), all of which had positive meanings. The questionnaire obtained a Cronbach's alpha coefficient equal to 0.82 using a 5 level rating scale.

(iv) The CIP. It consisted of a self-management program combined with the social support, heart healthy eating diet guide for CVD patients, learning plans, and teaching materials (power points) that correspond to the content of the CIP. In summary, the CIP consisted of the following.

(A) Goal Selection: This comprised a set of criteria that were developed to achieve the goals and measure success. The patient and family joined the nurse or the research team in determining the criteria that each person was assessed against to evaluate the success after receiving information about the disease or illness.

(B) Information collection: This is the procedure that the patients must perform and monitor themselves. Self-monitoring is conducted by self-observation and self-recording. Patients must observe and record their self-management, their eating behavior, preferred food menu, and an awareness of the sources of social support (may come from relatives, care-givers, or medical personnel) to use for information in determining the next objective.

(C) Information analysis and evaluation (Information process and evaluation): This is the use of the collected data to compare with the specified criteria. This may be the standard or specific value of each patient. Patients and family, the research term or a primary care nurse gathered together to discuss about the data and evaluation for the next step.

(D) Decision making: This is the most important step because after thoroughly analyzing the data, the patient must be able to make an appropriate decision about when or how he or she will manage the activity or behavior.

(E) Action: This refers to an act to manage self-health care; that is, the patients control and adjust their eating behavior according to the knowledge that they received, including self-management skills, to arrange a good food menu, to know how to use nutrition therapy, to be aware of social support healthcare providers and the other benefits of continuous care systems.

(F) Self-reaction: This is an assessment of the ability of the patient's confidence level. In selfmanagement practices, to achieve the goals based on their self-reaction assessment, the patients will have to be able to anticipate the actual action or to practice the required skills in order to obtain the proper eating behaviors.

The questionnaires on the patient's eating behavior and self-management assessment were $1^{\text {st }}$ piloted using 30 patients similar to the sample group, which gave a calculated reliability of the questionnaires of 0.90 and 0.92 , respectively.

\section{Ethical considerations}

Before launching the study, ethical approval was obtained from the research ethics review committee for research involving human research participants, health sciences group, Chulalongkorn University, Thailand. (Certified code: 064.1/60). In addition, a written consent form was obtained from each participant. Patients in the study were reassured about the anonymity and confidentiality of any obtained information. They were also informed about their right to withdraw from the study at any time.

\section{Data analysis}

Demographic and illness factors were analyzed by frequency distribution, percentage, mean and standard deviation (SD). Comparison of the differences in the average healthy eating behavior scores and self-management scores of the CVD patients before and after participation in the CIP were made using a dependent t-test, while those between the post-CIP group and the control CVD group were compared using an independent t-test. 


\section{Results and discussion}

From table 1 revealed the demographic and illness characteristics were not significantly different between the participants in the CIP and control groups, with respect to their age, gender, marital status, occupation, comorbidity, BMI, and history of treatment (all $\mathrm{p}>0.05$ ). In the experimental group the average age was 55.7 year, a slight majority were male (65\%), marital status were pair (75\%), the main employment category was in agriculture $(40.1 \%)$, and most had at least one other disease. with an average BMI of $52.3 \%$ (range 23.0 - $24.9 \%$ ), They had been treated by percutaneous cardiac intervention once $(35.7 \%)$, twice $(56.9 \%), 3$ times $(8.6 \%)$, and CABG $(6.0)$.

In the control group, the average age was 50.5 year, a slight majority were male $(65 \%)$, marital status were pair (70\%), the main employment category was in agriculture $(35.0 \%)$, and most had at least 1 other disease. with an average BMI of $48.2 \%$ (range $23.0-24.9 \%$ ), They had been treated by percutaneous cardiac intervention once (30.9\%), twice (48.3\%), 3 times (15.8\%), and CABG (5.0).

Table 1 The demographic and illness characteristics of the study participants

\begin{tabular}{lll}
\hline & Experimental group $(\mathbf{n}=\mathbf{2 5})$ & Control group $(\mathbf{n}=\mathbf{2 5})$ \\
\hline Mean age (year) & 55.7 & 50.5 \\
Age range & $40-65$ & $40-62$ \\
Gender (\%) & & \\
man & 65.0 & 65.0 \\
woman & 45.0 & 45.0 \\
Marital status & & \\
Pair & 75.0 & 70.0 \\
Single & 25.0 & 30.0 \\
Occupation (\%) & & \\
Police & 25.0 & 28.0 \\
Agriculture & 40.0 & 35.0 \\
Employment & 20.0 & 14.0 \\
No work & 15.0 & 23.0 \\
Co-morbidity (\%) & & \\
1 - 3 & 65.0 & 60.0 \\
$3-6$ & 3.05 & 40.0 \\
BMI & & \\
$<18.5$ & - & - \\
18.5 - 22.9 & - & - \\
23.0 - 24.9 & 52.3 & 48.2 \\
25.0 - 29.9 & 34.2 & 40.6 \\
$>30$ & 13.2 & 11.2 \\
History of treatment & & \\
PCI (Once) & 34.7 & 30.9 \\
PCI (Twice) & 50.7 & 48.3 \\
PCI ( 3 times) & 8.6 & 15.8 \\
CABG & 6.0 & 5.0 \\
\hline
\end{tabular}

Effect of the self-management program combined with a social support

The average scores of the self-management and healthy eating behavior questionnaires of the CVD patients in the CIP group before and after participation in the CIP are summarized in Table 2. 
Table 2 Comparison of the average score for CVD patients before and after participation in the CIP compared to the control (no CIP participation) group.

\begin{tabular}{|c|c|c|c|c|}
\hline \multirow[t]{2}{*}{ Group } & \multicolumn{2}{|c|}{ Self-management } & \multicolumn{2}{|c|}{ Healthy eating behavior } \\
\hline & Before & After & Before & After \\
\hline \multicolumn{5}{|c|}{ Experimental group $(n=25)$} \\
\hline $\begin{array}{l}\text { Mean } \pm \text { SD } \\
\text { p-value }\end{array}$ & $\begin{array}{c}46.2 \pm 5.6 \\
<0.05\end{array}$ & $51.1 \pm 5.9$ & $\begin{array}{c}39.3 \pm 5.5 \\
<0.05\end{array}$ & $48.4 \pm 4.0$ \\
\hline $\begin{array}{l}\text { Cor } \\
\text { Mean } \pm \text { SD } \\
\text { p-value }\end{array}$ & $47.0 \pm 6.3$ & $48.9 \pm 6.0$ & $\begin{array}{r}37.1 \pm 7.3 \\
0\end{array}$ & $39.8 \pm 7.5$ \\
\hline
\end{tabular}

From Table 2, the CIP group showed a significant increase in the average score of both their selfmanagement and, especially, in the healthy eating behaviors after participating in the CIP compared to before, while the control group showed a slight numerical increase in their scores but this was not significant for either the self-management or healthy eating behavior scores. There was no significant difference between the experimental (CIP) group before participation in the CIP and the control group at either time point

Table 3 Comparison of the average score for the healthy eating behavior and self-management questionnaires between CVD patients in the CIP group after treatment with the control group who received regular nursing care.

\begin{tabular}{llccc}
\hline Variable & Mean \pm SD & t & df & p-value \\
\hline Self-management & & & & \\
Experimental group $(\mathrm{n}=25)$ & $51.1 \pm 5.9$ & 4.25 & 48 & $<0.05$ \\
$\quad$ Control groups $(\mathrm{n}=25)$ & $48.9 \pm 6.0$ & & & \\
Healthy eating behaviors & & & & \\
Experimental group $(\mathrm{n}=25)$ & $48.4 \pm 4.0$ & 2.96 & 48 & \\
$\quad$ Control groups $(\mathrm{n}=25)$ & $39.8 \pm 7.5$ & & & \\
\hline
\end{tabular}

From Table 3, the CIP group after participation in the CIP scored significantly higher in both the self-management or healthy eating behavior questionnaires than the control group.

\section{Discussion}

The current study hypothesized that firstly, CVD patients exposed to the CIP would have a greater improvement in their self-management and positive healthy eating behaviors indices than before the course, and secondly, to the CVD patients on just the usual nursing care. The results supported both hypotheses, where significant improvements in both the patients' positive self-management and healthy eating behaviors average scores were found in the CIP group after attending the CIP compared to before it and those for the patients in the control group. The 2 groups of patients involved in the study were similar in most of their socio-demographic characteristics and their self-management and healthy eating 
behaviors (pre-test). So the changes obtained after the CIP were not attributed to differences in these characteristics between the groups nor simply to anything in the 6-week duration of non-CIP treatment.

Factors related to healthy eating behaviors were the difficulties in changing personal habits, having limited access to healthy food sources, food prices, difficulty in food preparation, limited knowledge of CVD and the benefits of healthy food, and the family's attitude towards healthy food in the CVD risk groups $[8,9,28,29]$. Accordingly, the knowledge adopted in the self-management program combined with the social support included information on CVD, knowledge and skills in organizing specific menu items, nutrition therapy, and food selection were based on cultural belief. These were the crucial points to improve knowledge, understanding about CVD and proper behavior. In addition, the support from family, health providers with information, knowledge, and various service systems will help patients become more confident and able to manage themselves effectively, change the diet habit, and maintain good (healthy) eating behaviors.

The result could be described in terms of good self-management. Good self-management will lead to better eating behavior or dietary habits following the concept of the self-management model. This is combined with motivational and self-regulatory skills to promote healthful lifestyles by enabling programs that supplant sedentariness with an active life. It can also foster the adoption of healthy diet behavior, which can aid in controlling BMI. For each health habit, people are manipulated to improve their health functioning, monitor their health habits, goal setting, short and long-term, and report the changes and feedback of progress toward their sub-goals. The effectiveness in feedback also guides how to manage the problem. This made the participants set new sub-goals to realize if beneficial changes are to be achieved and maintained in behavior $[14,17,30]$.

Self-management of patients is the act of patients solving problems on their own [30] and includes goal selection, information collection, information processing and evaluation, decision making, action, and self-reflection (self-reaction) [16]. This process improves the patient's self-management ability, confidence in themselves and can change their eating behaviors to be healthier. Therefore, the selfmanagement and healthy eating behavior scores were significantly higher in the CIP group after completing the 6-week program.

These findings agree with a previous study that evaluated the effects of behavioral management programs on blood lipid levels in patients with post-coronary intervention; in that the self-efficacy score and diet behavior increased in the experimental group while the total cholesterol HDL increased but the LDL level decreased [27]. Likewise, after receiving a supportive and educative nursing program, the patients' diet behavior scores were reported to be higher than before joining the program [31]. In contrast, CHD patients showed increased average scores in their healthy eating behavior after receiving the health intervention, although their serum lipid profile did not change [12].

In addition to improving the patients' self-management to adjust and maintain their healthier eating behaviors, social support was also adopted in this program through the 3 support dimensions of emotional, tangible, and information support [17,18]. The social support in this study included family, spouse, and personnel in the health care system. Effective social support would help motivate the patient and their spouse to take care of each other, make each other feel confident, pay attention to the kind of food eaten, and search for further dietary knowledge [12]. Thus, the average self-management and healthy eating behavior scores of patients in the CIP groups were significantly higher than in the control group.

The result of this study is in accordance with that previously found for CVD patients who were supported by a health personnel team. These patients were able to adjust their eating habits more easily than those who were not supported by health personnel [21]. Receiving support, encouragement, and advice from families, spouses, and friends helped the patients choose more suitable foods for their health and better eating habits $[20,21,32,33]$. Therefore, the family is centered and is the most effective in enhancing CVD patients' personal health [34,35].

An important issue to be considered in this program is the systematically maintained care for patients. The follow-up system, both by phone and home visits, in coordination with government health agencies responsible for the area and research team was adopted in this CIP. The health personnel's home visit helped the patients reflect, assess, and solve their problems and evaluate their self-management ability and behavior changes. It created a trusting and good relationship (doctor-patient \& family 
relationship) in which the process of learning and holistic care by the patient center can be established. The system for continuous patient care, coordination, and assistance can be developed in the same way throughout the health system. Then, patients would be more satisfied and willing to cooperate in self-care, change and maintain good behaviors, and obtain a higher QOL.

\section{Conclusions}

Patients with CVD after joining the CIP (self-management program combined with social support) had a higher self-confidence or self-management ability, which was manifested as significantly higher average scores in both their self-management and healthy eating behaviors than in the CVD patients receiving only the usual nursing care. The self-management program focused on providing patients with 6 different steps: Goal selection, information collection, information processing and evaluation, decision making action, and self-reflection (self-reaction) by themselves. The social support from relatives, healthcare providers, family, and spouse helped to change and maintain the changes in self-management and healthy eating behaviors.

\section{Recommendations}

Future studies should consider the health care system and mobile technologies towards a system for continuous patient care, where coordination and assistance can be developed in the same way throughout the health system. Applications on a mobile phone are one type of assistant for closely following the behaviors or clinical signs that need immediate solutions, for example, chest pain, heart attack, forgetting to take the necessary medicine, and so on. Therefore, patients remain satisfied and willing to cooperate in self-care, change to and maintain healthier behaviors, and will be able to live under CVD with a better QOL, while the rate of hospitalization with complications will also be decreased.

\section{Acknowledgements}

This research was supported by the National Research University Project, Office of Higher Education Commission. The authors thank the Graduate School, Chulalongkorn University for support in research assistance of fund, participants, and the professional medical and primary care nurse at Bang Nam Priao Hospital, Chachoengsao province (Thailand), for conducting the research and all information.

\section{References}

[1] B Remenyi, N Wilson, A Steer, B Ferreira, J Kado, K Kumar, J Lawrenson, G Maguire, E Marijon, M Mirabel, AO Mocumbi, C Mota, J Paar, A Saxena, J Scheel, J Stirling, S Viali, VI Balekundri, G Wheaton, L Zühlke and J Carapetis. World heart federation criteria for echocardiographic diagnosis of rheumatic heart disease: An evidence-based guideline. Nat. Rev. Cardiol. 2012; 9, 297 309.

[2] World Health Organization. Cardiovascular diseases (CVDs) Available at: http://www.who.int/ mediacentre/factsheets/fs317/en/index.html, accessed 2011.

[3] AS Go, D Mozaffarian, VL Roger, EJ Benjamin, JD Berry, WB Borden, DM Bravata, S Dai, ES Ford, CS Fox, S Franco, HJ Fullerton, C Gillespie, SM Hailpern, JA Heit, VJ Howard, MD Huffman, BM Kissela, SJ Kittner, DT Lackland, JH Lichtman, LD Lisabeth, D Magid, GM Marcus, A Marelli, DB Matchar, DK McGuire, ER Mohler, CS Moy, ME Mussolino, G Nichol, NP Paynter, PJ Schreiner, PD Sorlie, J Stein, TN Turan, SS Virani, ND Wong, D Woo and MB Turner. Heart disease and stroke statistics-2013 update: A report from the American Heart Association. Circulation 2013; 127, e6-e245.

[4] SM Mosleh and M Darawad. Patients' adherence to healthy behavior in coronary heart disease: Risk factor management among Jordanian patients. J. Cardiovasc. Nurs. 2015; 30, 471-8. 
[5] IM Lee, EJ Shiroma, F Lobelo, P Puska, SN Blair, and PT Katzmarzyk. Effect of physical inactivity on major non-communicable diseases worldwide: An analysis of burden of disease and life expectancy. Lancet 2012; 380, 219-29.

[6] B Aggarwal, M Liao, A Christian, and L Mosca. Influence of caregiving on lifestyle and psychosocial risk factors among family members of patients hospitalized with cardiovascular disease. J. Gen. Intern. Med. 2009; 24, 93-8.

[7] LG Martinez and MA House-Fancher. Medical surgery nursing: Assessment and management of clinical problem. $9^{\text {th }}$ ed. Mosby, Missouri, 2000.

[8] L Barandon, J Calderon, M Lafitte, L Leroux, JL Cadusseau, X Roques and T Couffinhal. Assessing cardiovascular risk factors after coronary artery bypass surgery: Value of an aggressive strategy including systematic follow-up. Arch. Cardiovasc. Dis. 2008; 101, 155-62.

[9] TT Fung, KM Rexrode, CS Mantzoros, JE Manson, WC Willett and FB Hu. Mediterranean diet and incidence of and mortality from coronary heart disease and stroke in women. Circulation 2009; 119, 1093-100.

[10] A Mente, LD Koning, HS Shannon and SS Anand. A systematic review of the evidence supporting a causal link between dietary factors and coronary heart disease. Arch. Intern. Med. 2009; 169, 65969.

[11] C Na-Sungchan. 2010, Self-care behavioral in patients after percutaneous cardiac intervention. Master Thesis. KhonKaen University, Khonkaen, Thailand.

[12] BC Yates, J Norman, J Meza, KS Krogstrand, S Harrington, S Shurmur, M Johnson and $\mathrm{K}$ Schumacher. Effects of partners together in health intervention on physical activity and healthy eating behaviors: A pilot study. J. Cardiovasc. Nurs. 2015; 30, 109-20.

[13] W Lehakun. Nutrition in nursing. Boonsiri Printing, Bangkok, Thailand, 2002, p. 406.

[14] TL Creer. Self-management of chronic illness. In: M Boekaerts, PR Pintrich and M Zeidner (Eds.). Handbook of self-regulation academic. Academic Press, San Diego, p. 601-29.

[15] JS House, D Umberson and KR Landis. Structures and processes of social support. Annu. Rev. Sociol. 1988; 14, 293-318.

[16] S Cohen, LG Underwood and Gottlieb BH. Social support measurement and intervention: A guide for health and social scientists. Oxford University Press, New York, 2000, p. 345.

[17] A Bandura. Health promotion from the perspective of social cognitive theory. In: $\mathrm{P}$ Norman, C Abraham and M Conner (Eds.). Understanding and changing health behaviour: From health beliefs to self-regulation. Harwood Academic, Amsterdam, 2000, p. 299-339.

[18] M Stewart, K Davidson, D Meade, A Hirth and P Weld-Viscount. Group support for couples coping with a cardiac condition. J. Adv. Nurs. 2001; 33, 190-9.

[19] A Bunmeesrisup. 2009, Factor predicting/food consumption behaviors in patient with cardiovascular disease. Master Thesis. Mahidol University, Bangkok, Thailand.

[20] DA Wood, K Kotseva, S Connolly, C Jennings, A Mead, J Jones, A Holden, DD Bacquer, T Collier, GD Backer, O Faergeman and EUROACTION Study Group. Nurse-coordinated multidisciplinary, family-based cardiovascular disease prevention programme (EUROACTION) for patients with coronary heart disease and asymptomatic individuals at high risk of cardiovascular disease: A paired, cluster-randomised controlled trial. Lancet 2008; 371, 1999-2012.

[21] CY Bernice, N Joseph, M Jane, SK Kaye, H Susana, S Scott, M Johnson and K Schumacher. Effects of partners together in health intervention on physical activity and healthy eating behaviors. J. Cardiovasc. Nurs. 2015; 30, 109-20.

[22] DF Polit and CT Beck. Nursing research: Principles and methods. Lippincott Williams \& Wilkins, Philadelphia, 2004

[23] N Burns and SK Grove. The practice of nursing research: Conduct, critique, and utilization. $6^{\text {th }}$ ed. WB Saunders Company, London, 2009.

[24] JH Hibbard, ER Mahoney, J Stockard and M Tusler. Development and testing of a short form of the patient activation measure. Health Serv. Res. 2005; 40, 1918-30. 
[25] P Kanokor. 2017, The effect of the self-management program on functional ability of patients with lumbar spine surgery in rehabilitation. Master Thesis. Chulalongkorn University, Bangkok, Thailand.

[26] DG Schlundt, MK Hargreaves and MS Buchowski. The eating behavior patterns questionnaire predicts dietary fat intake in African American women. J. Am. Diet. Assoc. 2003; 103, 338-45.

[27] T Pachanat. 2010, The effect of behavioral management program on blood cholesterol in patients with coronary revascularization. Ph. D. Dissertation. Chulalongkorn University, Bangkok, Thailand.

[28] CK Chow, S Jolly, P Rao-Melacini, KA Fox, SS Anand and S Yusuf. Association of diet, exercise, and smoking modification with risk of early cardiovascular events after acute coronary syndromes. Circulation 2010; 121, 750-8.

[29] NT Artinian, GF Fletcher, D Mozaffarian, P Kris-Etherton, L Van Horn, AH Lichtenstein, S Kumanyika, WE Kraus, JL Fleg, NS Redeker, JC Meininger, J Banks, EM Stuart-Shor, BJ Fletcher, TD Miller, S Hughes, LT Braun, LA Kopin, K Berra, LL Hayman, LJ Ewing, PA Ades, JL Durstine, N Houston-Miller, LE Burke and Prevention Committee of the Council on Cardiovascular Nursing. Interventions to promote physical activity and dietary lifestyle changes for cardiovascular risk factor reduction in adults: A scientific statement from the American Heart Association. Circulation 2010; 122, 406-41.

[30] R Kanfer and PL Ackerman. Aging, adult development, and work motivation. Acad. Manag. Rev. 2004; 29, 440-58.

[31] P Khongseua. The effects of educative supportive nursing program on dietary behavior among patients after percutaneous transluminal coronary intervention. Kuakarun J. Nurs. 2014; 21, 168-85.

[32] AH Lichtenstein, LJ Appel, M Brands, M Carnethon, S Daniels, HA Franch, B Franklin, P KrisEtherton, WS Harris, B Howard, N Karanja, M Lefevre, L Rudel, F Sacks, LV Horn, M Winston and J Wylie-Rosett. Diet and lifestyle recommendations revision 2006: A scientific statement from the American Heart Association Nutrition Committee. Circulation 2006; 114, 82-96.

[33] SC SmithJr, EJ Benjamin, RO Bonow, LT Braun, MA Creager, BA Franklin, RJ Gibbons, SM Grundy, LF Hiratzka, DW Jones, DM Lloyd-Jones, M Minissian, L Mosca, ED Peterson, RL Sacco, J Spertus, JH Stein and KA Taubert. AHA/ACCF secondary prevention and risk reduction therapy for patients with coronary and other atherosclerotic vascular disease: 2011 update: A guideline from the American Heart Association and American College of Cardiology Foundation. Circulation 2011; 124, 2458-73.

[34] LC Macken, B Yates and S Blancher. Concordance of risk factors in female spouses of male patients with coronary heart disease. J. Cardiopulm. Rehabil. 2000; 20, 361-8.

[35] L Mosca, H Mochari, M Liao, AH Christian, DJ Edelman, B Aggarwal and MC Oz. A novel family-based intervention trial to improve heart health: FIT Heart: Results of a randomized controlled trial. Circ. Cardiovasc. Qual. Outcomes 2008; 1, 98-106. 